\title{
Interplay of surface reconstruction and surface electric fields in the optical anisotropy of $\operatorname{GaAs}(001)$
}

\author{
W. G. Schmidt and F. Bechstedt \\ Institut für Festkörpertheorie und Theoretische Optik, Friedrich-Schiller-Universität, Max-Wien-Platz 1, D-07743 Jena, Germany \\ W. Lu and J. Bernholc \\ Department of Physics, North Carolina State University, Raleigh, North Carolina 27695-8202
}

(Received 16 April 2002; published 30 August 2002)

\begin{abstract}
The optical anisotropy of $c(4 \times 4),(2 \times 4),(2 \times 6)$ and $(4 \times 2)$ reconstructed GaAs $(001)$ surfaces has been calculated from first principles. It consists of surface structure-dependent features originating from electronic transitions in the uppermost surface layers and of anisotropy peaks close to the $E_{1}$ and $E_{0}^{\prime} / E_{2}$ bulk critical point energies. The latter contributions are nearly structure independent and arise from transitions between surface-modified bulk electronic states. For the smaller reconstructions the influence of surface electric fields on the optical anisotropy is studied. We find that the linear electro-optic effect modifies mainly the optical anisotropy from the bulk atomic layers, resulting in changes of the reflectance anistropy spectroscopy signal which are strongly reconstruction dependent, however. Changes of the atomic relaxation due to surface electric fields are less important for the modification of the optical signal than the polarization of the electron wave functions.
\end{abstract}

DOI: 10.1103/PhysRevB.66.085334

\section{INTRODUCTION}

Optical spectroscopies, in particular reflectance anisotropy spectroscopy (RAS), often termed reflectance difference spectroscopy (RDS), have become very important for in situ characterization of static surfaces and real-time monitoring of surface growth. Aspnes and Studna ${ }^{1}$ discriminate between two components of surface optical spectra: "intrinsic" contributions arising from optical transitions within the bulk and "extrinsic" contributions directly related to surface chemistry. The latter can often be traced to specific surface electronic states and serve as fingerprints for surface structural motifs. ${ }^{2-4}$

The GaAs(001) surface with its large number of stoichiometry-dependent surface reconstructions and its importance for III-V based optoelectronics is a very popular model system for the application of RAS. Nevertheless, the origin of its optical anisotropy features is still not understood. Positive anisotropies close to the $E_{1}$ and $E_{0}^{\prime} / E_{2}$ bulk critical point $(\mathrm{CP})$ energies, for example, are commonly interpreted as fingerprints for the formation of As dimers. ${ }^{5-9} \mathrm{In}$ contrast, very recent experiments on GaAs surface quantum wells indicate that these peaks are intrinsic rather than extrinsic features of the surface optical spectrum. ${ }^{10}$ Previous $a b$ initio calculations ${ }^{11,12}$ could not clarify the origin of these features unequivocally, due to the computational difficulties to calculate numerically converged surface optical spectra.

The actual origin of the intrinsic RAS features is another intriguing question. For a long time they have been assumed to be related mainly to surface local fields, ${ }^{13,14}$ i.e., the influence of the surface-modified microscopic fluctuations of the electromagnetic field on the macroscopic dielectric response. However, $a b$ initio calculations on $\mathrm{Si}(110): \mathrm{H}$ surfaces ${ }^{15}$ showed recently that the influence of local fields on the optical anisotropy may actually be rather small. Surfaceinduced strain and relaxation in bulk layers were discussed as another possible reason for intrinsic RAS features. ${ }^{16,17}$
PACS number(s): 78.68.+m, 78.66.Fd, 73.20.At

First-principles calculations for $\mathrm{Si}(001)$ (Ref. 18) indicated, however, that these factors modify, rather than drastically alter, the optical anisotropy signals.

Surface electric fields may be an alternative explanation. Related to the changed surface dipole, the ionization energy of the GaAs surface varies as a function of the surface composition and, thereby, reconstruction. ${ }^{19}$ For example, the measured ionization energies of the $\alpha, \beta$, and $\gamma$ phases of the $\operatorname{GaAs}(001)$ surface amount to $5.4,5.5$, and $5.2 \mathrm{eV}$, respectively. ${ }^{20}$ Similar values are obtained from $a b$ initio calculations. ${ }^{21}$ Due to the linear electro-optic effect, some influence of the reconstruction-dependent surface dipole on the RAS can be expected. Experimentally it has been known for a long time that electric fields induced by, e.g., the pinning of the Fermi level at the sample surface ${ }^{22-25}$ or by a $\delta$-doping layer ${ }^{26}$ modify the RAS signal. The investigation of the mechanism behind the electric-field-induced modification of the optical signal may not only help to better understand the origin of the intrinsic RAS features, but should pave the way for applications, such as contactless determination of the carrier concentration in a bulk material. Possible mechanisms for explaining the influence of electric fields on the surface optical response are shifts of the surface state energies, ${ }^{27}$ the piezoelectric effect, ${ }^{28,29}$ and the polarization of the electron wave functions. ${ }^{30}$ Model calculations based on the piezoelectric effect ${ }^{28,29}$ successfully described the electric field induced line shape changes around the $E_{1}$ and $E_{1}+\Delta_{1} \mathrm{CP}$ energies. However, the influence of the surface reconstruction on the field-induced RAS changes or wider spectral ranges have not been considered.

Here we present first-principles calculations of the optical anisotropy of the main GaAs(001) surface reconstructions, shown in Fig. 1. The contributions to the spectra are classified according to their spatial origin into bulk and surface features. In addition, we calculate the influence of a surface electric field ranging from -0.015 to $0.015 \mathrm{~V} / \AA$ on the RAS spectra of the $c(4 \times 4),(2 \times 4)$, and $(4 \times 2)$ reconstructed surfaces. 


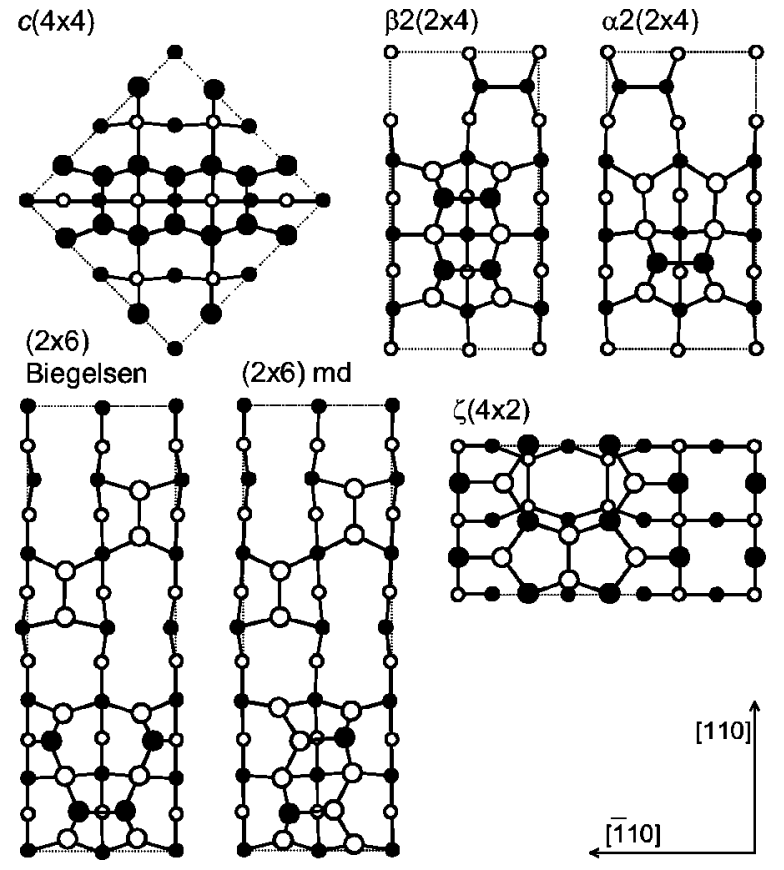

FIG. 1. Top view of relaxed $\mathrm{GaAs}(001)$ surface structures. Empty (filled) circles represent Ga (As) atoms. Positions in the uppermost two atomic layers are indicated by larger symbols.

\section{COMPUTATIONAL METHOD}

Our density-functional (DFT-LDA) calculations are based on a real-space multigrid implementation using nonlocal pseudopotentials. ${ }^{31}$ The electron wave functions are mapped on a grid with a spacing corresponding to $4 \%$ of the GaAs bulk lattice constant. The surface is modeled by periodic super cells containing 12 atomic (001) layers and a vacuum region 8 atomic layers thick. Further details of the DFT-LDA calculations are those in Refs. 3,32. The electronic structure obtained within DFT-LDA is used to calculate the surface optical anisotropy ${ }^{33,34}$ in the independent-particle approximation. In general, optical spectra are strongly modified by many-body effects such as self-energy corrections and electron-hole attraction. ${ }^{15,35-37}$ However, RAS spectra are difference spectra, which are furthermore normalized to the bulk dielectric function. Due to the error cancellation, singleparticle calculations within DFT-LDA are actually quite reliable in predicting surface optical anisotropies. ${ }^{3}$ Therefore, and because of the large number of optical spectra calculated in the present work, we simply use the scissors-operator approach $^{38}$ to take self-energy effects into account. Excitonic and local-field effects are neglected. A saw-tooth function added to the electrostatic potential entering the Kohn-Sham equations is used to mimic the effect of an electric field perpendicular to the crystal surface. From the self-consistent solution of the Kohn-Sham equations the influence of the electric field on both the wave functions and the eigenvalues is obtained. Additionally, the surface atomic geometry in the presence of an electric field is recalculated in case of the $c(4 \times 4)$ reconstruction. The sign convention used here is such that the field points in the direction of the surface normal.

\section{RESULTS AND DISCUSSION}

\section{A. Surface-structure influence on the RAS}

The stoichiometry-dependent surface structures of GaAs(001) (see Fig. 1) have been studied intensively over the last decade. ${ }^{39}$ There now seems to be consensus on the geometries of the As-rich $c(4 \times 4)$ and $\beta 2(2 \times 4)$ reconstructions, as well as on the $\alpha 2(2 \times 4)$ structure occurring for stoichiometric surfaces. ${ }^{32,39,40}$ Little, however, is known about the geometry of the $(2 \times 6)$ reconstructed surface, often observed in conjunction with other $(n \times 6)$ symmetries. $^{39,41,42}$ We model the $(2 \times 6)$ surface by the structure proposed by Biegelsen et al. ${ }^{43}$ This structure represents a stoichiometric surface. The preparation conditions typical for the appearance of $(n \times 6)$ symmetries, however, are cation rich. ${ }^{39,42}$ Anion-cation heterodimers are formed on many cation-rich III-V(001) surfaces. ${ }^{40}$ Therefore, we also investigate the replacement of the top-layer As dimers by mixed Ga-As dimers. Both Biegelsen's model and the mixed-dimer model for the $(2 \times 6)$ surface are only metastable and should therefore only be observed as transient structures. Finally, the Ga-rich $(4 \times 2)$ surface is modeled in the present work by the $\zeta$ structure, favored by recent $a b$ initio calculations ${ }^{32,44}$ and supported by $\mathrm{x}$-ray diffraction experiments. $^{45,46}$

In Fig. 2 the RAS spectra calculated for the surface models discussed above are shown. Obviously, the surface optical anisotropy is strongly related to the surface geometry. The spectra calculated for $c(4 \times 4)$ and $\beta 2(2 \times 4)$ agree well with experiment ${ }^{47-49}$ (some experimental curves from Ref. 47 are redrawn in Fig. 3). The appearance of negative anisotropies for the $\alpha 2(2 \times 4)$ structure at low photon energies also agrees with the experimentally observed trend: annealing temperatures higher than those needed to prepare the $\operatorname{GaAs}(001) \beta 2(2 \times 4)$ surface lead to negative anisotropies at about $2 \mathrm{eV}^{47,48}$ This minimum in the optical anisotropy is also found at $\mathrm{InP}$ and $\mathrm{GaP}(001)$ surfaces for the corresponding structures. ${ }^{2,51}$ It originates from electronic transitions involving the cation-cation surface bonds. However, in the GaAs experiment (see Fig. 3) the positive anisotropy at the $E_{1}$ energy does not disappear, as calculated here for the $\alpha 2$ model. This may be explained by the transient character of the $\alpha 2$ structure, which in case of $\mathrm{GaAs}(001)$ is stable only for a very small window of preparation conditions. ${ }^{40}$ Therefore, it should occur together with other surface geometries, such as $\beta 2$.

RAS spectra measured for $(n \times 6)$ surfaces $^{42,47}$ show a pronounced minimum at about $2 \mathrm{eV}$. In addition, positive anisotropies at the $E_{0}^{\prime} / E_{2} \mathrm{CP}$ energies are observed. The anisotropies around the $E_{1}$ energy are strongly preparation dependent. The reflectance anisotropy calculated for Biegelsen's $(2 \times 6)$ model is positive for all considered photon energies. Therefore, it cannot explain the experimental findings. Together with the Ga-rich preparation conditions, which can hardly be reconciled with the stoichiometric Biegelsen model, our results thus indicate that another structure is responsible for the experimental observation of $(n \times 6)$ symmetries. The spectrum calculated for the $(2 \times 6)$ mixeddimer model features negative anisotropies around $2 \mathrm{eV}$, to- 

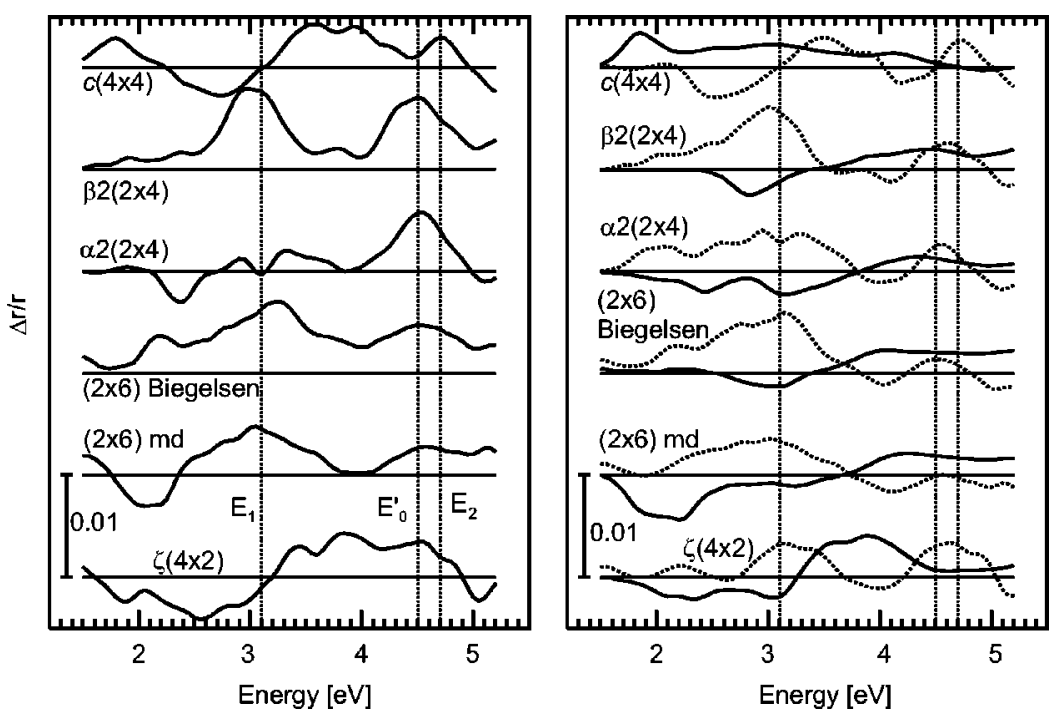

FIG. 2. Calculated RAS spectra $\left[\operatorname{Re}\left\{\left(r_{[1 \overline{1} 0]}\right.\right.\right.$ $\left.\left.\left.-r_{[110]}\right) /\langle r\rangle\right\}\right]$ for GaAs(001) surface reconstructions shown in Fig. 1. The left panel shows the total signal, whereas solid/dotted lines in the right panel show separately the contributions of surface/bulk layers to the RAS. The calculated positions of the bulk $\mathrm{CP}$ energies are indicated. gether with positive anisotropies at the $E_{0}^{\prime} / E_{2} \mathrm{CP}$ energies. The main experimental features are thus reproduced, suggesting mixed Ga-As dimers as one possible building block for $(n \times 6)$ reconstructed $\mathrm{GaAs}(001)$ surfaces.

The main experimentally observed RAS features for Garich $(4 \times 2)$ reconstructed surfaces are negative anisotropies for photon energies below the $E_{1} \mathrm{CP}$ and positive anisotropies between the $E_{1}$ and $E_{0}^{\prime}$ energies (see Fig. 3). This is roughly reproduced by our calculation for the $\zeta(4 \times 2)$ surface. The agreement between theory and experiment, however, is not as good as for the As-rich surfaces. This holds in particular for the comparison with the low-temperature data of Ref. 49. The discrepancies may be related to surface defects: a recent X-ray analysis by Kumpf et $a l .{ }^{45}$ suggests that $19 \%$ of the $(4 \times 2)$ surface unit cells are decorated with additional $\mathrm{Ga}$ adatoms.

Although the RAS is strongly dependent on the surface structure, as discussed above, not only transitions between surface states contribute to the spectra. By means of a linear cutoff function applied to the transition matrix elements cal-

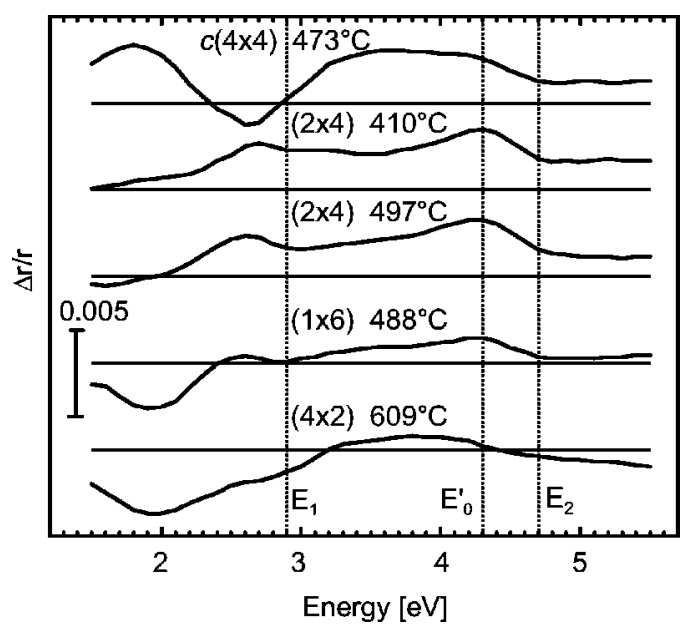

FIG. 3. RAS spectra $\left[\operatorname{Re}\left\{\left(r_{[1 \overline{1} 0]}-r_{[110]}\right) /\langle r\rangle\right\}\right]$ measured for GaAs(001) surface reconstructions (after Ref. 47). The measured positions of the bulk CP energies at $673 \mathrm{~K}$ (Ref. 50) are indicated. culated in real space, one can roughly divide the RAS in bulk and surface contributions. Such a division is shown in the right panel of Fig. 2, where we have separated the contributions from the uppermost four layers from the signals induced by transitions in the layers underneath. The latter are only weakly structure dependent, as is the case for other III-V(001) surfaces. ${ }^{3}$ Surface modified bulk electronic states give rise to positive signals around the $E_{1}$ and $E_{0}^{\prime} / E_{2}$ bulk $\mathrm{CP}$ energies for nearly all surface models. For the $\beta 2(2$ $\times 4$ ) structure, the reflectance anisotropy is almost entirely determined by transitions between surface-modified bulk electronic states. This is in contrast to the tight-binding results by Murayama and co-workers, ${ }^{6,7}$ who associated the reflectance anisotropy near the $E_{1}$ energy with As-dimer related surface states. Berkovits and co-workers ${ }^{5}$ also interpreted the weakening of the $E_{1}$ feature upon adsorption of oxygen as indication of its relation to As-dimer states. Such a conclusion is not imperative, however. A simple cancellation of bulk- and surface-related optical anisotropies, such as calculated here for the $\alpha 2$ structure, might be responsible. A very recent experiment by Lastras-Martinez et al. ${ }^{10}$ clearly supports our prediction of the bulk origin of the $E_{1}$ feature in the $\beta 2$ spectrum.

\section{B. Electric-field-induced changes of the RAS signal}

In order to clarify to what extent the RAS features are modified or caused by surface electric fields we subject our slab to an external electric field perpendicular to the crystal surface. The field polarizes the electron wave functions, slightly changes the density of states, and in addition leads to small structural changes. In Fig. 4 we show the RAS calculated for the $\mathrm{GaAs}(001) c(4 \times 4)$ under the influence of an electric field of $\pm 0.015 \mathrm{~V} / \AA$. Dotted lines refer to the equilibrium geometry of the surface ground state, as calculated in the absence of additional electric fields. The solid lines represent the RAS calculated for surfaces, where the equilibrium atomic positions have been adjusted according to the additional forces due to the electric field. The differences between the two sets of curves are small, of the order of the 


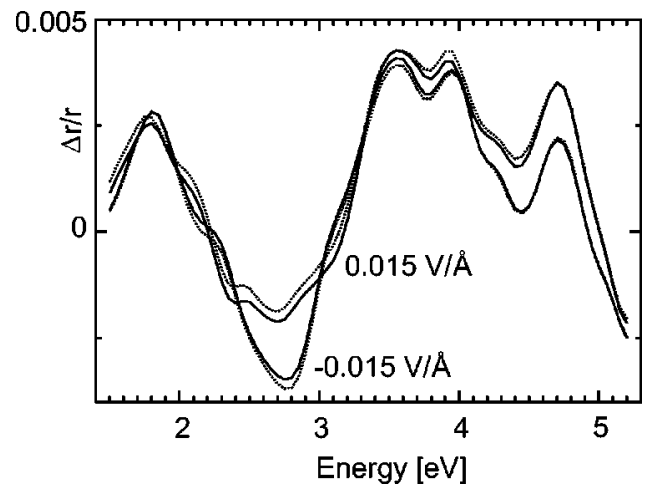

FIG. 4. Calculated RAS spectra for $\operatorname{GaAs}(001) c(4 \times 4)$ with an applied electric field of $\pm 0.015 \mathrm{~V} / \AA$. Solid (dotted) lines refer to calculations where the field-induced atomic relaxations are (not) considered.

purely numerical accuracy of our calculations. This clearly indicates that the electric-field-induced distortions of the electron wave functions have a far greater influence on the optical anisotropy than the relaxation of the lattice in response to the field. We therefore neglect field-induced lattice relaxation effects in the following.

The calculated RAS spectra of $c(4 \times 4), \beta 2(2 \times 4)$, $\alpha 2(2 \times 4)$, and $\zeta(4 \times 2)$ reconstructed $\mathrm{GaAs}(001)$ surfaces for fields ranging from -0.015 to $0.015 \mathrm{~V} / \AA$ are shown in Fig. 5. In all cases the magnitude and to some extent the
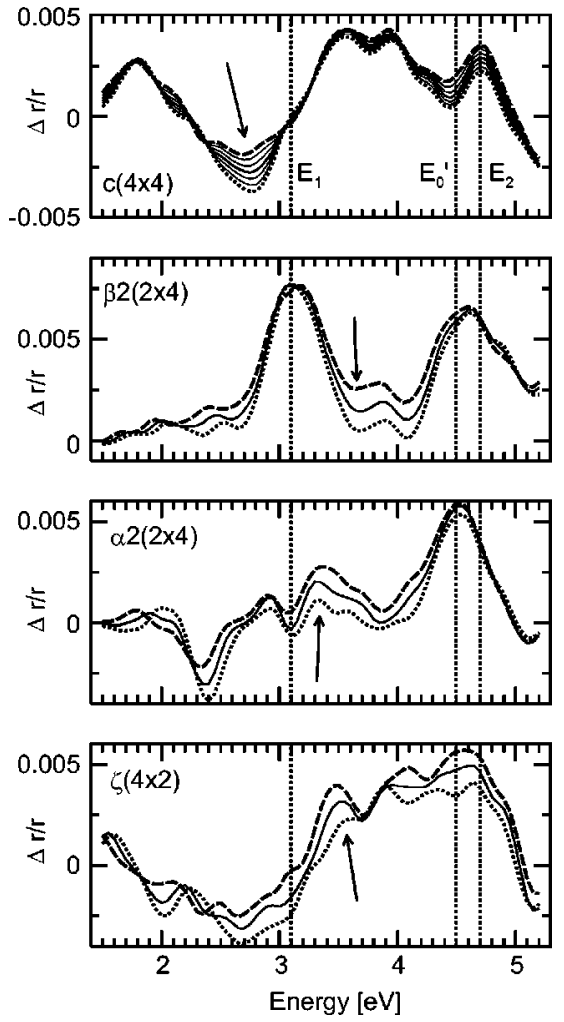

FIG. 5. Calculated RAS spectra for GaAs(001) surface reconstructions with electric fields applied. For $\operatorname{GaAs}(001) c(4 \times 4)$ the field varies in steps of $0.005 \mathrm{~V} / \AA$. Thick solid/dotted/dashed lines correspond the field magnitudes of $0.0 /-0.015 / 0.015 \mathrm{~V} / \AA$. energy positions of specific RAS features are modified. A remarkable outcome of our study is the strong reconstruction dependence of the electric-field-induced RAS changes. For the $c(4 \times 4)$ reconstructed GaAs surface, strong modifications of the RAS signal are observed for photon energies below $3 \mathrm{eV}$ and at the $E_{0}^{\prime} / E_{2} \mathrm{CP}$ energies. The optical anisotropy of the ( $2 \times 4)$ reconstructed $\beta 2$ and $\alpha 2$ surfaces, on the other hand, is mainly altered in the energy region between the $E_{1}$ and $E_{0}^{\prime}$ CPs. Finally, nearly the entire RAS spectrum of the Ga-rich $\zeta(4 \times 2)$ surface is modified by the application of an electric field. Our finding of a strong influence of the surface reconstruction on the field-induced RAS is supported by recent experiments: for photon energies below the $E_{1} \mathrm{CP}$, Pristovsek et al. ${ }^{52}$ found larger differences of the RAS of $c(4 \times 4)$ and $(4 \times 2)$ reconstructed surfaces of doped and undoped GaAs samples than for $(2 \times 4)$ reconstructions. On the other hand, the RAS of the $(2 \times 4)$ surface was found to be more sensitive to electric fields for photon energies above the $E_{1}$ energy.

Although the influence of the electric field on the surface optical anisotropy is reconstruction dependent, we find that it is mainly the signal from the bulk layers that is modified. This is obvious in case of the $c(4 \times 4)$ reconstructed surface. The strongest change of the RAS signal occurs at the minimum around $2.8 \mathrm{eV}$. This minimum is due to optical transitions in the bulk layers, as shown in Fig. 2. The spatial analysis of the origin of the RAS features shows also for the other surface structures that it is the optical signal from the layers underneath the surface, i.e., the intrinsic anisotropy, that is affected by the field. Our calculations thus support the view that the surface optical anisotropy for photon energies near the bulk CP's is a consequence of the decay of the bulk Bloch states into the vacuum region, anisotropically modified by the surface potential. The surface-induced deformations of the bulklike wave functions are weighted differently by the $x$ and $y$ components of the optical transition operator, leading to an anisotropic optical response. Superimposed on the surface-induced anisotropies of the electron wave functions are the electric-field-induced deformations. These are anisotropic too, as visualized in Fig. 6 for the electron density of the $\operatorname{GaAs}(001) \beta 2(2 \times 4)$ surface slab. The electric field influences both the electron wave functions and their eigenvalues. We find, however, that the changes of the combined density of states due to the electric field are negligible compared to the deformations of the wave functions resulting in modified transition matrix elements.

Our results on the influence of electric fields on the optical anisotropy of $\mathrm{GaAs}(001)$ surfaces cannot directly be compared with experiment. On one hand, this is due to computational shortcomings. We neglect the spin-orbit coupling and therefore cannot describe the $E_{1} / E_{1}+\Delta_{1}$ splitting. The RAS features close to the $E_{1} / E_{1}+\Delta_{1}$ energy are considerably affected by the spin-orbit interaction. Most measurements of the linear electro-optic effect focus specifically on these features. On the other hand, the comparison of experimental and simulated spectra is complicated by the facts that (i) the magnitude of the surface electric field, induced by, e.g., $\delta$ doping or space-charge layers is not known exactly 


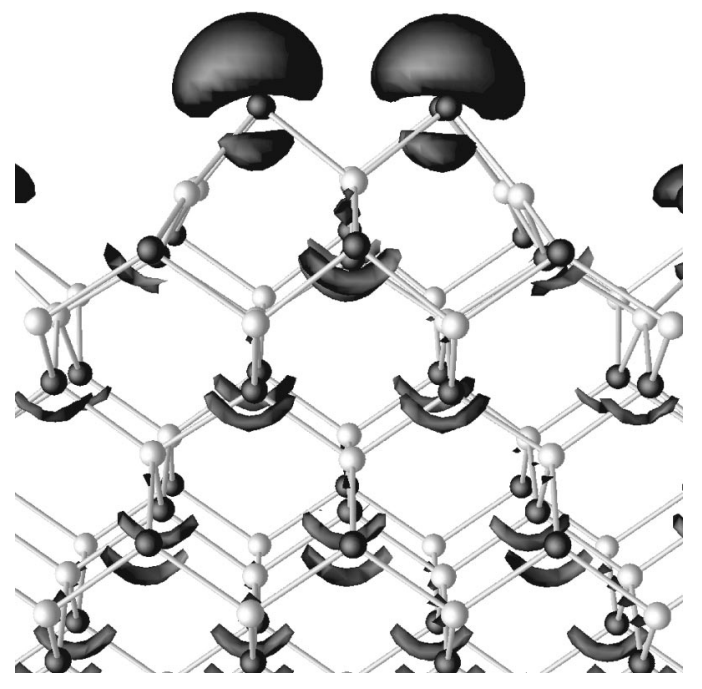

FIG. 6. Electron density transfer (isosurfaces $\Delta \rho$ $=10^{-4} e / \mathrm{Bohr}^{3}$ ) induced by an electric field of $0.015 \mathrm{~V} / \AA$ in a slab modeling the $\operatorname{GaAs}(001) \beta 2(2 \times 4)$ surface. Empty (filled) circles represent $\mathrm{Ga}(\mathrm{As})$ atoms.

and (ii) most experiments are performed on surfaces, the geometries of which are not well characterized, e.g., oxidized surfaces.

In measurements done in air on $\mathrm{GaAs}(001)$ surfaces, Yang, Chen and Wong ${ }^{53}$ found a linear relationship between the change in the RAS at the $E_{1} / E_{1}+\Delta_{1}$ energy and the surface electric field. They determined a linear electro-optic coefficient of $0.46 \AA$ /V. In Fig. 7 we show the change of the RAS signal for the four surface reconstructions at energy positions indicated in Fig. 5, i.e., slightly below or above the $E_{1}$ transition as a function of the electric field. Clearly, the calculated change of the RAS is linear, as observed experimentally. The slopes of the curves in Fig. 7 are about 0.07 $\AA / \mathrm{V}$, much smaller than the measured value of $0.46 \AA / \mathrm{V}$. The

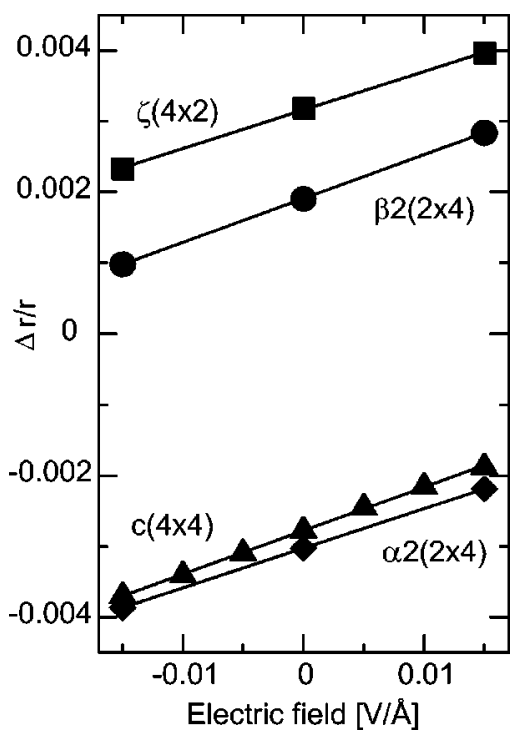

FIG. 7. Variation of the RAS calculated for GaAs(001) surface reconstructions at the photon energies marked by arrows in Fig. 5, for applied electric fields. The solid lines are a guide to the eye. fact that the field needed in the calculation to reproduce changes in the RAS comparable in magnitude to experiment is nearly one order of magnitude stronger than measured, is related to the large penetration depth of light. It amounts to about $170 \AA$ for a photon energy of $3 \mathrm{eV},{ }^{54}$ whereas our slab only models the uppermost $15 \AA$ of the GaAs surface. By varying the distance between the $\delta$-doping layer and the surface Sobiesierski, Westwood, and Elliot ${ }^{26}$ have shown that the surface electric field reaches far into the bulk and modifies the optical response along the full light penetration depth. The incoming light decreases in intensity as it penetrates the sample, therefore the ratio between the light penetration depth and the slab thickness gives an upper bound for the scaling factor needed to relate the calculated and the measured field sensitivity of the RAS. The value of the electro-optic coefficient calculated here is thus in the expected range. This agreement may be fortuitous, however, given the neglect of spin-orbit coupling in our study. Nevertheless, because both experiment and calculations find a linear relation between the changes of the optical anisotropy and the surface electric field, we expect our results, although obtained for comparatively strong fields, to correctly describe the basic mechanisms responsible for the field-induced RAS changes.

\section{SUMMARY}

We have investigated the effects of reconstructions and surface electric fields on the optical anisotropy of GaAs(001) from first principles. Our results for As-rich $c(4 \times 4)$ and $(2 \times 4)$ as well as Ga-rich $(4 \times 2)$ reconstructions are in good agreement with experiment. The line shape measured for $(n \times 6)$ reconstructed surfaces, on the other hand, cannot be reproduced by calculations based on Biegelsen's model for the $(2 \times 6)$ reconstructed surface. A trial structure, containing mixed Ga-As dimers, rather than anion dimers, leads to a spectrum in much better agreement with experiment. This indicates that novel structural motifs might be needed to explain the $(n \times 6)$ symmetries of GaAs. Apart from surfacestructure signatures, the RAS spectra contain contributions from electronic transitions between surface-perturbed bulk states that depend only weakly on the surface reconstruction. Mainly the latter, intrinsic features are modified by surface electric fields via the linear electro-optic effect. The electricfield-induced modifications of the RAS features are strongly reconstruction dependent. They do not overwhelm, however, the RAS signals due to the anisotropy of the surface itself. The field-induced changes in the RAS signal are caused by the polarization of the electron wave functions rather that by field-induced lattice relaxations.

\section{ACKNOWLEDGMENTS}

We thank Dave Aspnes, Markus Pristovsek, and Norbert Esser for many useful discussions. Grants of computer time from the DoD Challenge Program, the LeibnizRechenzentrum München, the Höchstleistungsrechenzentrum Stuttgart and the John von Neumann-Institut Jülich are gratefully acknowledged. This work was supported in part by ONR and DoE. 
${ }^{1}$ D.E. Aspnes and A.A. Studna, Phys. Rev. Lett. 54, 1956 (1985).

${ }^{2}$ W.G. Schmidt, N. Esser, A.M. Frisch, P. Vogt, J. Bernholc, F. Bechstedt, M. Zorn, T. Hannappel, S. Visbeck, F. Willig, and W. Richter, Phys. Rev. B 61, R16 335 (2000).

${ }^{3}$ W.G. Schmidt, F. Bechstedt, and J. Bernholc, J. Vac. Sci. Technol. B 18, 2215 (2000).

${ }^{4}$ W. Lu, W.G. Schmidt, E.L. Briggs, and J. Bernholc, Phys. Rev. Lett. 85, 4381 (2000).

${ }^{5}$ V.L. Berkovits, P. Chiaradia, D. Paget, A.B. Gordeeva, and C. Goletti, Surf. Sci. 441, 26 (1999).

${ }^{6}$ M. Murayama, K. Shiraishi, and T. Nakayama, Jpn. J. Appl. Phys. 36, L268 (1997).

${ }^{7}$ M. Murayama, K. Shiraishi, and T. Nakayama, Jpn. J. Appl. Phys. 37, 4109 (1998).

${ }^{8}$ M.J. Begarney, L. Li, C.H. Li, D.C. Law, Q. Fu, and R.F. Hicks, Phys. Rev. B 62, 8092 (2000).

${ }^{9}$ Y.-C. Chang and D.E. Aspnes, Phys. Rev. B 41, 12002 (1990).

${ }^{10}$ L.F. Lastras-Martinez, D. Rönnow, P.V. Santos, M. Cardona, and K. Eberl, Phys. Rev. B 64, 245303 (2001).

${ }^{11}$ R. Eryiğit and I.P. Herman, Phys. Rev. B 56, 9263 (1997).

${ }^{12}$ S.J. Morris, J.M. Bass, and C.C. Matthai, Phys. Rev. B 52, 16739 (1995).

${ }^{13}$ W.L. Mochán and R.G. Barrera, Phys. Rev. Lett. 55, 1192 (1985).

${ }^{14}$ B.S. Mendoza, R. Del Sole, and A.I. Shkrebtii, Phys. Rev. B 57, R12 709 (1998).

${ }^{15}$ P.H. Hahn, W.G. Schmidt, and F. Bechstedt, Phys. Rev. Lett. 88, 016402 (2002).

${ }^{16}$ K. Hingerl, R.E. Balderas-Navarro, W. Hilber, A. Bonanni, and D. Stifter, Phys. Rev. B 62, 13048 (2000).

${ }^{17}$ K. Hingerl, R.E. Balderas-Navarro, A. Bonanni, P. Tichopadek, and W.G. Schmidt, Appl. Surf. Sci. 175, 769 (2001).

${ }^{18}$ W.G. Schmidt, F. Bechstedt, and J. Bernholc, Phys. Rev. B 63, 045322 (2001).

${ }^{19}$ W. Mönch, Semiconductor Surfaces and Interfaces (SpringerVerlag, Berlin, 1995).

${ }^{20}$ H. Tsuda and T. Mizutani, Appl. Phys. Lett. 60, 1570 (1992).

${ }^{21}$ W.G. Schmidt and F. Bechstedt, Phys. Rev. B 54, 16742 (1996).

${ }^{22}$ V.L. Berkovits, I.V. Makarenko, T.A. Minashvili, and V.I. Safarov, Sov. Phys. Semicond. 20, 654 (1986).

${ }^{23}$ S.E. Acosta-Ortiz and A. Lastras-Martinez, Solid State Commun. 64, 809 (1987).

${ }^{24}$ S.E. Acosta-Ortiz and A. Lastras-Martinez, Phys. Rev. B 40, 1426 (1989).

${ }^{25}$ M. Pristovsek, S. Tsukamoto, N. Koguchi, B. Han, K. Haberland, J.T. Zettler, W. Richter, M. Zorn, and M. Weyers, Phys. Status Solidi A 188, 1423 (2001).

${ }^{26}$ Z. Sobiesierski, D.I. Westwood, and M. Elliott, Phys. Rev. B 56, 15277 (1997).

${ }^{27}$ K.-M. Ho, B.N. Harmon, and S.H. Liu, Phys. Rev. Lett. 44, 1531 (1980).
${ }^{28}$ Y.H. Cheng and Z. Yang, Appl. Phys. Lett. 73, 1667 (1998).

${ }^{29}$ A. Lastras-Martinez, R.E. Balderas-Navarro, L.F. LastrasMartinez, and M.A. Vidal, Phys. Rev. B 59, 10234 (1999).

${ }^{30}$ B.A. Foreman, Phys. Rev. Lett. 84, 2505 (2000).

${ }^{31}$ E.L. Briggs, D.J. Sullivan, and J. Bernholc, Phys. Rev. B 54, 14362 (1996).

${ }^{32}$ W.G. Schmidt, S. Mirbt, and F. Bechstedt, Phys. Rev. B 62, 8087 (2000).

${ }^{33}$ R. Del Sole, Solid State Commun. 37, 537 (1981).

${ }^{34}$ F. Manghi, R. Del Sole, A. Selloni, and E. Molinari, Phys. Rev. B 41, 9935 (1990).

${ }^{35}$ S. Albrecht, L. Reining, R. Del Sole, and G. Onida, Phys. Rev. Lett. 80, 4510 (1998).

${ }^{36}$ M. Rohlfing and S.G. Louie, Phys. Rev. Lett. 83, 856 (1999).

${ }^{37}$ L.X. Benedict, E.L. Shirley, and R.B. Bohn, Phys. Rev. Lett. 80, 4514 (1998).

${ }^{38}$ R. Del Sole and R. Girlanda, Phys. Rev. B 48, 11789 (1993).

${ }^{39}$ Q.-K. Xue, T. Hashizume, and T. Sakurai, Prog. Surf. Sci. 56, 1 (1997).

${ }^{40}$ W.G. Schmidt, Appl. Phys. A: Mater. Sci. Process. 75, 89 (2002).

${ }^{41}$ J.G. McLean, P. Kruse, and A.C. Kummel, Surf. Sci. 424, 206 (1999).

${ }^{42}$ M. Pristovsek, S. Tsukamoto, A. Ohtake, N. Koguchi, B. Orr, W. Schmidt, and J. Bernholc, Surf. Sci. (to be published).

${ }^{43}$ D.K. Biegelsen, R.D. Bringans, J.E. Northrup, and L.E. Swartz, Phys. Rev. B 41, 5701 (1990).

${ }^{44}$ S.-H. Lee, W. Moritz, and M. Scheffler, Phys. Rev. Lett. 85, 3890 (2000).

${ }^{45}$ C. Kumpf, L.D. Marks, D. Ellis, D. Smilgies, E. Landemark, M. Nielsen, R. Feidenhans'1, J. Zegenhagen, O. Bunk, J.H. Zeysing, Y. Su, and R.L. Johnson, Phys. Rev. Lett. 86, 3586 (2001).

${ }^{46}$ D. Paget, Y. Garreau, M. Sauvage, P. Chiaradia, R. Pinchaux, and W.G. Schmidt, Phys. Rev. B 64, 161305 (2001).

${ }^{47}$ I. Kamiya, D.E. Aspnes, L.T. Florez, and J.P. Harbison, Phys. Rev. B 46, 15894 (1992).

${ }^{48}$ A.I. Shkrebtii, N. Esser, W. Richter, W.G. Schmidt, F. Bechstedt, B.O. Fimland, A. Kley, and R. Del Sole, Phys. Rev. Lett. 81, 721 (1998).

${ }^{49}$ W. G. Schmidt, F. Bechstedt, K. Fleischer, C. Cobet, N. Esser, W. Richter, J. Bernholc, and G. Onida, Phys. Status Solidi A 188, 1401 (2001).

${ }^{50}$ P. Lautenschlager, M. Garriga, S. Logothetidis, and M. Cardona, Phys. Rev. B 35, 9174 (1987).

${ }^{51}$ W.G. Schmidt, J.L. Fattebert, J. Bernholc, and F. Bechstedt, Surf. Rev. Lett. 6, 1159 (1999).

${ }^{52}$ M. Pristovsek and S. Tsukamoto, Bull. Am. Phys. Soc. 47, 85 (2002); M. Pristovsek, S. Tsukamoto, B. Han, J. -T. Zettler, and W. Richter, J. Cryst. Growth (to be published).

${ }^{53}$ Z. Yang, Y.H. Chen, and Y. Wong, Appl. Phys. Lett. 73, 1520 (1998).

${ }^{54}$ D.E. Aspnes and A.A. Studna, Phys. Rev. B 27, 985 (1983). 